\title{
Chronic pain assessment: A seven-factor model
}

\author{
Megan A Davidson MA ${ }^{1}$, Dean A Tripp PhD², Leandre R Fabrigar PhD ${ }^{1}$, Paul R Davidson PhD ${ }^{1}$
}

\begin{abstract}
MA Davidson, DA Tripp, LR Fabrigar, PR Davidson. Chronic
pain assessment: A seven-factor model. Pain Res Manage
MA Davidson, DA Tripp, LR Fabrigar, PR Davidson. Chronic
pain assessment: A seven-factor model. Pain Res Manage 2008;13(4):299-308.
\end{abstract}

BACKGROUND: There are many measures assessing related dimensions of the chronic pain experience (eg, pain severity, pain coping, depression, activity level), but the relationships among them have not been systematically established.

OBJECTIVE: The present study set out to determine the core dimensions requiring assessment in individuals with chronic pain.

METHODS: Individuals with chronic pain $(n=126)$ completed the Beck Anxiety Inventory, Beck Depression Inventory, Beck Hopelessness Scale, Chronic Pain Coping Index, Multidimensional Pain Inventory, Pain Catastrophizing Scale, McGill Pain Questionnaire - Short Form, Pain Disability Index and the Tampa Scale of Kinesiophobia.

RESULTS: Before an exploratory factor analysis (EFA) of the nine chronic pain measures, EFAs were conducted on each of the individual measures, and the derived factors (subscales) from each measure were submitted together for a single EFA. A seven-factor model best fit the data, representing the core factors of pain and disability, pain description, affective distress, support, positive coping strategies, negative coping strategies and activity.

CONCLUSIONS: Seven meaningful dimensions of the pain experience were reliably and systematically extracted. Implications and future directions for this work are discussed.

Key Words: Affective distress; Chronic pain assessment; Coping; Disability; Factor analysis

\section{Évaluation de la douleur chronique: Modèle à sept facteurs}

\begin{abstract}
HISTORIQUE : Plusieurs outils de mesure existent pour évaluer différentes dimensions interdépendantes de la douleur chronique (p. ex., intensité de la douleur, mécanismes d'adaptation, dépression, degré d'activité), mais leurs liens n'ont jamais été établis de manière systématique. OBJECTIF : La présente étude vise à déterminer les principales dimensions de la douleur chronique qu'il convient d'évaluer chez les personnes atteintes.

MÉTHODES : Des personnes qui souffrent de douleur chronique $(n=126)$ ont répondu à divers questionnaires : inventaires d'anxiété, de dépression et de désespoir de Beck, indice d'adaptation à la douleur chronique, inventaire multidimensionnel de la douleur, échelle de catastrophisation de la douleur, échelle McGill de mesure de la douleur-SF, indice d'incapacité liée à la douleur, et échelle de kinésiophobie de Tampa.

RÉSULTATS : Avant une analyse factorielle exploratoire (AFE) des neuf échelles de mesure de la douleur chronique, des AFE individuelles ont été réalisées sur chacune d'entre elles et leurs facteurs dérivés respectifs (sous-échelles) ont été soumis ensemble à une AFE regroupée. C'est un modèle à sept facteurs qui s'adapte le mieux aux données, représentant les principales dimensions suivantes : douleur et incapacité, description de la douleur, détresse émotionnelle, soutien, stratégies d'adaptation positives, stratégies d'adaptation négatives et activité.

CONCLUSIONS : Sept dimensions significatives de l'expérience de la douleur ont pu être extraites de manière fiable et systématique. On décrit également la portée et les développements futurs des présents travaux.
\end{abstract}

\section{CHRONIC PAIN: UNDERLYING CONSTRUCTS}

Pain is a complex perceptual experience, influencing and influenced by a wide range of factors, including biological systems, emotions such as depression and anxiety, social and environmental contexts, pain appraisal, beliefs, attitudes and expectations (1). As such, an appropriate assessment of individuals suffering from chronic pain must evaluate these multiple dimensions. Although some of the measures used in pain assessment are observational (eg, behavioural coding of facial expression during pain), many are self-report measures. Most of the psychometric studies of self-report measures used in chronic pain assessment have been limited to investigations of individual measures. For the most part, such measures have been demonstrated to be reliable and valid, showing clear relationships with other important chronic pain variables. However, few studies have examined chronic pain assessment measures in aggregate. There is inadequate information regarding the extent to which different measures capture the same construct, and the extent to which they capture a unique construct. Thus, researchers and clinicians cannot be certain how many different constructs or factors are assessed by standard measures used in chronic pain assessment.

To date, two studies $(2,3)$ have examined the factors underlying chronic pain assessment measures. Using exploratory factor analysis (EFA), Mikail et al (2) identified five factors underlying their set of 36 subscales derived from nine chronic pain measures. These factors included general affective distress, coping, support, pain description and functional capacity. Based on their analyses, Mikail et al selected three measures comprising 14 subscales that, they argued, best captured the pain experience. These measures included the Beck Depression Inventory (BDI) (4), the McGill Pain Questionnaire (MPQ) (5) and the Multidimensional Pain Inventory (MPI) (6). In a subsequent study, De Gagne et al (3) extended this work using confirmatory factor analysis (CFA) to test Mikail et al's model. Their analyses used the 14 subscales put forth by Mikail et al.

\footnotetext{
${ }^{1}$ Department of Psychology; ${ }^{2}$ Departments of Psychology, Anesthesiology and Urology, Queen's University, Kingston, Ontario

Correspondence: Megan A Davidson, Department of Psychology, Queen's University, Humphrey Hall, 62 Arch Street, Kingston, Ontario

K7L 3N6. Telephone 613-533-6000 ext 75677, fax 613-533-2499, e-mail 9md9@queensu.ca
} 
De Gagne et al's results indicated a four-factor solution: affective distress, support, pain description and functional capacity (pain and disability). These results supported the work of Mikail et al; DeGagne et al found four of the same five factors underlying their measures. The fifth factor, coping, did not emerge in the second study, because no measure of coping behaviour was included.

The work of Mikail et al (2) and De Gagne et al (3) represents a solid beginning in understanding the nature of the relationships among chronic pain measures. From this small body of research, a number of important factors underlying chronic pain assessment can be seen, including affective distress, support, pain and disability, and coping. However, these studies have limitations, and more work is needed to clarify these factors.

One important limitation of these previous studies pertains to the methodology used to derive the number of factors. Factor analysis represents a collection of different techniques, and researchers have a choice in the type of model-fitting procedures (eg, principal factors, maximum likelihood) and the type of rotation they use (eg, oblique versus orthogonal). Furthermore, there are a number of procedures available to determine the appropriate number of factors. Some of these procedures have been criticized as being highly problematic (eg, eigenvalues greater than one), whereas others are likely to perform reasonably well (eg, parallel analysis, descriptive indexes of model fit such as root mean square error of approximation [RMSEA]) (7). In addition, in determining the appropriate number of factors, it has been suggested that researchers rely on multiple criteria. Fabrigar et al (7) noted that on their own, even the best factordetermining procedures are not infallible.

In their EFA, Mikail et al (2) determined the appropriate number of factors using a single criterion of retaining factors with eigenvalues greater than one (the Kaiser criterion). This method of determining the appropriate number of factors has been criticized for a number of reasons, including its tendency to be misapplied, its arbitrariness and its tendency to overfactor (or in some cases, underfactor) (7-9).

A second limitation pertains to the factor structures of the individual pain measures used. Both Mikail et al (2) and De Gagne et al (3) examined the factor structure of their measures together without first examining the factor structure of each of the individual measures. This approach is problematic. Before one can understand the underlying factors of the measures as a group, one must first understand the underlying factors of each individual measure. In the chronic pain literature, there is inconsistency among studies in the reported underlying factor structures of individual measures. For example, factoranalytic studies of the McGill Pain Questionnaire - Short Form (SF-MPQ) (10) have yielded two- (11) and three-factor (12) solutions. Factor-analyzing studies of the Pain Catastrophizing Scale (PCS) (13) have also yielded two- (14) and three-factor $(13,15)$ solutions. These inconsistencies may be due to differences in the factor-analytic techniques used. Such inconsistencies are problematic, because it is not clear which, if any, of the factor solutions of these measures represent appropriate or accurate divisions of items. Thus, because De Gagne et al and Mikail et al failed to examine the factor structure of each of the individual measures before examining the measures together, their analyses may have produced imprecise or inaccurate results.

The present study was designed to improve the investigation of the factors underlying chronic pain measures by addressing the limitations of earlier research. There was a need for a more comprehensive examination of chronic pain assessment measures using more rigourous methodology. We examined the inter-relationships among nine measures commonly used in chronic pain assessment. These included the SF-MPQ, Pain Disability Index (PDI) (16), Tampa Scale of Kinesiophobia (TSK) (17), PCS, Chronic Pain Coping Index (CPCI) (18), MPI, BDI, Beck Hopelessness Scale (BHS) (19) and the Beck Anxiety Inventory (BAI) (20). These measures were selected primarily because of their clinical use in the tertiary care pain clinic setting for guiding the development of individualized clinical interventions. Furthermore, the purpose of the present study was to examine the factors underlying the chronic pain experience, including its antecedents and consequences. Thus, it was important to include a wide range of measures of chronic pain beyond self-reported pain and disability. The measures examined in the present study are similar to those used by Mikail et al (2) and De Gagne et al (3). However, the pain assessment literature has advanced since their research, and we have included new measures that have appeared in the chronic pain literature (eg, CPCI, PCS, TSK) to reflect these advances.

In addition to the inclusion of updated measures, the present study used a sample comprising both community-living and clinic-referred individuals with chronic pain. The two previous factor analyses used samples of individuals with chronic pain referred to multidisciplinary pain clinics. By increasing the heterogeneity of our sample, the results of the present study were intended to be generalizable to a larger group of individuals with chronic pain. Finally, the present study improved on previous studies by using more rigorous methodology to determine the factor structure underlying these measures together. Before an EFA of the nine chronic pain measures together, EFAs were conducted on the items comprising each of the individual measures. Then, the derived factors from each of the nine measures were submitted together for a single EFA. In addition, for all factor analyses, we used multiple criteria to determine the appropriate number of factors.

\section{METHODS}

\section{Participants}

Participants included 126 community-living individuals with chronic pain (40 men, 86 women). Participants ranged in age from 16 to 91 years, with a mean ( \pm SD) age of $50.38 \pm 14.21$ years. The sample was similar in age and sex to other chronic pain samples (21). Data were collected from two samples: one of individuals with chronic pain referred to an outpatient pain clinic between 2003 and 2005 ( $n=65 ; 16$ men, 49 women), the other of individuals with chronic pain identified through an epidemiological survey (22) ( $n=61 ; 24$ men, 37 women). Chronic pain in participants from the epidemiological sample was identified using the Graded Chronic Pain Survey (23). All participants spoke English. Inclusion criteria were chronic pain (pain for longer than six months), and the ability to read and write. Participants were excluded if they did not experience chronic pain.

Participants in the current study were primarily Caucasian $(91.3 \%)$, with a small number of Pacific Islanders and Native Hawaiians (2.4\%), African Americans (1.6\%), Asians (0.8\%), Aboriginals (0.8\%) and Hispanics (0.8\%). This distribution of ethnicities is typical of the area sampled (Kingston, Ontario) (24). Employment status varied: $34.9 \%$ were unemployed, 
$31.7 \%$ were disabled, $19.8 \%$ were retired and $11.9 \%$ were employed. The education level of the sample also varied: $27.0 \%$ had completed a college or university program, $24.6 \%$ had completed some college or university; $19.8 \%$ had a high school or general equivalency diploma, $13.5 \%$ had some high school education, and $8.7 \%$ had attended a graduate or professional school. With respect to marital status, $59.5 \%$ of participants reported that they were married, $19.0 \%$ were single, $8.7 \%$ were divorced, $4.0 \%$ were living with a common-law spouse, $2.4 \%$ were separated and $3.2 \%$ were widowed.

\section{Measures}

SF-MPQ: The SF-MPQ is a self-report measure of pain quality consisting of 15 descriptors of pain, representing both the sensory (eg, 'throbbing', 'aching') and affective (eg, 'sickening', 'fearful') components of pain quality. Participants are asked to indicate the extent to which each descriptor describes the severity of their pain experience. Responses are made on a four-point Likert scale, ranging from 0 (none) to 3 (severe). Three subscale scores are calculated: sensory, affective and total pain responses. Internal consistency estimates range from 0.73 to 0.89 (12) and test-retest reliability estimates range from 0.88 to 0.96 for each of the subscales (25). Reliability analyses with data from the present study indicated acceptable internal consistency estimates for all scales of the SF-MPQ (Cronbach's alphas were $0.87,0.81$ and 0.76 for the total, sensory and affective scales, respectively).

PDI: The PDI is a seven-item self-report measure that assesses disability in seven areas: family, occupation, sexual relations, social activities, recreation, self-care and life support. Participants are asked to indicate their disability in each of the seven areas. Responses are made on an 11-point Likert scale, ranging from 0 (no disability) to 10 (total disability). A total disability score is calculated. Internal consistency estimates are high (Cronbach's alpha $=0.86$ ) and item-total correlations range from 0.56 to $0.85(16,26)$. However, the only study to evaluate test-retest reliability showed it to be low $(r=0.44)$ (26). Reliability analyses of the PDI showed high internal consistency (Cronbach's alpha $=0.91)$.

TSK: The TSK is a 17 -item measure of the fear of movement, injury and reinjury (eg, "My body is telling me I have something dangerously wrong"; "It's not really safe for a person with a condition like mine to be physically active"). Responses are indicated on a four-point Likert scale, ranging from 1 (strongly disagree) to 4 (strongly agree). A total score is calculated. The TSK demonstrates adequate internal consistency (Cronbach's alphas range from 0.70 to 0.81$)$ and good test-retest reliability $(\mathrm{r}=0.78)(27-29)$. The instrument shows acceptable concurrent validity, with TSK scores correlating with other self-report measures of pain-related fear ( $r$ values range from 0.54 to 0.60 ) (27). Reliability analyses from the present study showed the TSK to have acceptable internal consistency (Cronbach's alpha $=0.79)$.

PCS: The PCS is a 13-item self-report measure of pain catastrophizing. Respondents are instructed to reflect on their pain experience and indicate the degree to which they experience thoughts or feelings during pain (eg, "When I'm in pain I worry all the time about when the pain will end"; "When I'm in pain I keep thinking about how much it hurts"). Each item is rated on a five-point Likert scale, ranging from 0 (not at all) to 4 (all the time). In addition to a full-scale pain catastrophizing score, three subscale scores are calculated: rumination, magnification and helplessness. Internal consistency values (Cronbach's alphas) for the full-scale PCS score range from 0.87 to 0.95 , and with the individual subscale scores, Cronbach's alphas range from 0.87 to 0.95 for rumination, 0.60 to 0.88 for magnification and 0.79 to 0.91 for helplessness $(13,14)$. Test-retest reliability of the measure is adequate ( $\mathrm{r}$ values range from 0.70 to 0.75 ) (13), and the scale demonstrates strong correlations with other measures of negative cognitions related to pain ( $\mathrm{r}$ values range from 0.53 to 0.59 ) (14). Reliability analyses from the present study showed the PCS to have acceptable internal consistency for all scales (Cronbach's alphas were $0.95,0.89,0.81$ and 0.89 for PCS total score, rumination, magnification and helplessness, respectively).

CPCI: The CPCI is a 65-item measure of cognitive and behavioural coping strategies often targeted as part of multidisciplinary pain treatment. Each item represents one coping strategy (eg, "Reminded myself that things could be worse"; "I rested as much as I could"). Respondents are asked to indicate the number of days they used the strategy to cope with pain over the previous week. Responses are indicated on an eightpoint Likert scale, ranging from 0 days to 7 days. Eight subscale scores are calculated, representing the use of different types of coping strategies: guarding, resting, asking for assistance, relaxation, task-persistence, exercise or stretching, seeking social support and coping self-statements. All subscales of the CPCI have been shown to be reliable, with internal consistencies ranging from 0.74 to 0.91 , and test-retest reliability greater than 0.70 (18). Reliability analyses from the present study showed acceptable internal consistency values for all of the eight CPCI scales (Cronbach's alphas ranging from 0.73 to 0.93).

MPI: The MPI is a 60-item measure designed to assess multiple aspects of psychosocial functioning in chronic pain patients. The measure consists of three sections, each with a number of subscales. Section I assesses pain impact and consists of five subscales: interference, support, pain severity, selfcontrol and negative mood. Section II assesses responses by significant others and produces three subscales: punishing responses, solicitous responses and distracting responses. Section III assesses activities and contains four subscales related to patients' activity levels: household chores, outdoor work, activities away from home and social activities. All subscales of the MPI demonstrate acceptable reliability, with Cronbach's alphas ranging from 0.69 to 0.92 for all 12 subscales (30). In the present study, reliability analyses of the scales produced varied results. Internal consistency was acceptable for 10 of the 12 scales (Cronbach's alphas ranged from 0.73 to 0.94 ); however, two scales from section III showed poor internal consistency (Cronbach's alphas were 0.61 and 0.69 for the activities away from home and social activities scales, respectively).

BDI: The BDI is a 21-item measure of depressive symptomatology, including items assessing both cognitive and somatic complaints associated with depression. Each item represents a symptom or belief that is rated from 0 to 3 in terms of intensity. The BDI consists of 21 groups of statements, and after reading each group of statements, participants mark the statement in each group that best describes the way they have been feeling over the previous week. One total depression score is generally calculated. Beck et al (31) performed a meta-analysis on 25 years of psychometric studies of the BDI using a variety of populations. Internal consistency estimates yielded a mean of 0.86 for 
TABLE 1

Scores for chronic pain measures

\begin{tabular}{lccc}
\hline & $\begin{array}{c}\text { Total } \\
\text { sample }\end{array}$ & $\begin{array}{c}\text { Clinic } \\
\text { sample }\end{array}$ & $\begin{array}{c}\text { Survey } \\
\text { sample }\end{array}$ \\
\hline BAI total & $14.47 \pm 10.59$ & $17.63 \pm 10.88$ & $11.52 \pm 9.70$ \\
BDI total & $16.95 \pm 10.73$ & $21.45 \pm 10.83$ & $11.86 \pm 8.82$ \\
BHS total & $7.36 \pm 5.63$ & $8.11 \pm 5.83$ & $6.33 \pm 5.22$ \\
CPCI guarding & $3.56 \pm 1.84$ & $3.84 \pm 1.70$ & $3.27 \pm 2.01$ \\
CPCI resting & $4.04 \pm 1.81$ & $4.44 \pm 1.72$ & $3.63 \pm 1.81$ \\
CPCI asking for assistance & $2.64 \pm 2.14$ & $3.19 \pm 2.18$ & $2.25 \pm 2.02$ \\
CPCI relaxation & $2.30 \pm 1.60$ & $2.56 \pm 1.69$ & $2.08 \pm 1.55$ \\
CPCI task persistence & $4.07 \pm 1.62$ & $3.56 \pm 1.46$ & $4.66 \pm 1.57$ \\
CPCI exercise/stretch & $2.47 \pm 2.06$ & $2.55 \pm 2.18$ & $2.46 \pm 2.10$ \\
CPCI seeking social support & $2.34 \pm 1.65$ & $2.64 \pm 1.66$ & $2.08 \pm 1.64$ \\
CPCI coping self-statements & $3.52 \pm 1.84$ & $3.89 \pm 1.79$ & $3.33 \pm 1.87$ \\
MPI pain severity & $4.04 \pm 1.26$ & $4.47 \pm 1.13$ & $3.54 \pm 1.24$ \\
MPI interference & $3.92 \pm 1.52$ & $4.53 \pm 1.25$ & $3.23 \pm 1.58$ \\
MPI life control & $3.31 \pm 1.41$ & $2.87 \pm 1.43$ & $3.85 \pm 1.21$ \\
MPI affective distress & $2.98 \pm 1.53$ & $3.51 \pm 1.41$ & $2.51 \pm 1.55$ \\
MPI support & $4.04 \pm 1.60$ & $4.43 \pm .156$ & $3.73 \pm 1.49$ \\
MPI punishing responses & $1.65 \pm 1.63$ & $1.63 \pm 1.77$ & $1.42 \pm 1.36$ \\
MPI solicitous responses & $3.05 \pm 1.62$ & $3.40 \pm 1.54$ & $2.69 \pm 1.58$ \\
MPI distracting responses & $2.20 \pm 1.38$ & $2.74 \pm 1.24$ & $1.73 \pm 1.36$ \\
MPI household chores & $3.76 \pm 1.62$ & $3.27 \pm 1.68$ & $4.14 \pm 1.54$ \\
MPI outdoor activities & $1.65 \pm 1.48$ & $1.11 \pm 1.29$ & $2.29 \pm 1.41$ \\
MPI activities away from home & $2.05 \pm 1.08$ & $1.80 \pm 0.99$ & $2.36 \pm 1.19$ \\
MPI social activities & $1.96 \pm 1.13$ & $1.87 \pm 1.17$ & $2.08 \pm 1.12$ \\
PCS total & $22.36 \pm 13.24$ & $27.23 \pm 11.79$ & $17.05 \pm 13.12$ \\
PCS rumination & $8.35 \pm 4.65$ & $9.87 \pm 4.11$ & $6.74 \pm 4.81$ \\
PCS magnification & $4.02 \pm 3.26$ & $4.77 \pm 3.22$ & $3.19 \pm 3.24$ \\
PCS helplessness & $9.99 \pm 6.30$ & $12.57 \pm 5.56$ & $7.12 \pm 6.09$ \\
PDI total & $35.68 \pm 18.52$ & $46.50 \pm 14.34$ & $24.05 \pm 16.35$ \\
SF-MPQ total & $21.12 \pm 9.69$ & $23.79 \pm 10.59$ & $18.40 \pm 8.86$ \\
SF-MPQ sensory & $16.17 \pm 7.09$ & $17.94 \pm 7.80$ & $14.37 \pm 6.65$ \\
SF-MPQ affective & $4.94 \pm 3.33$ & $5.85 \pm 3.73$ & $4.04 \pm 2.83$ \\
TSK & $39.66 \pm 8.91$ & $41.46 \pm 10.38$ & $37.54 \pm 7.36$ \\
\hline Rests & $+50.8 A 1$ & .19 & \\
\hline
\end{tabular}

Results presented as mean $\pm S D$. BAl Beck Anxiety Inventory; BDI Beck Depression Inventory; BHS Beck Hopelessness Scale; CPCI Chronic Pain Coping Inventory; MPI Multidimensional Pain Inventory; PCS Pain Catastrophizing Scale; PDI Pain Disability Index; SF-MPQ McGill Pain Questionnaire - Short Form; TSK Tampa Scale of Kinesiophobia

psychiatric inpatients and 0.81 for nonpsychiatric participants; test-retest correlations ranged from 0.48 to 0.86 . The authors also reported high correlations with clinical ratings of depression $(r=0.72)$, indicating good construct validity. Reliability analyses from the present study demonstrated high internal consistency (Cronbach's alpha $=0.92$ ).

BHS: The BHS is a 20-item measure of hopelessness. The measure consists of 11 negatively phrased (eg, "My future seems dark to me") and nine positively phrased items (eg, "I look forward to the future with hope and enthusiasm"). Responses are indicated using a true/false response format. A total hopeless score is calculated. Internal consistency estimates range from 0.88 to $0.93(19,32,33)$. Item-total correlations for the measure range from 0.39 to 0.76 in one study (19) and from 0.03 to 0.66 in a more recent psychometric investigation (33). Steed (33) also found strong evidence of convergent validity, with high correlations $(r=0.73$ to 0.79 ) between BHS scores and other measures of similar constructs. Analyses from the present study supported the reliability of the BHS, with high internal consistency (Cronbach's alpha = 0.92).

BAI: The BAI is a 21-item measure of anxiety severity. Each item represents one symptom of anxiety (eg, "numbness or tingling"; "fear of the worst happening") and responses are indicated on a four-point Likert scale, ranging from 0 (not at all) to 3 (severe, I could barely stand it). A total anxiety score is calculated. Investigations of psychometric characteristics of the BAI have demonstrated high internal consistency (Cronbach's alphas ranged from 0.90 to 0.92 ) and satisfactory test-retest reliability $(\mathrm{r}=0.75)(20,34)$. Convergent validity estimates indicate that the BAI is moderately and significantly correlated with other self-report anxiety scales ( $r=0.35$ to 0.69 ) (34). Data from the present study supported the reliability of the BAI, with high internal consistency (Cronbach's alpha = 0.91).

\section{Design and procedure}

The first sample of participants, comprising individuals referred to an outpatient chronic pain clinic, completed the assessment battery as part of their psychological assessment. All individuals who are referred to the clinic complete the battery of measures. Participants received the battery of measures in a fixed order (BAI, BDI, BHS, CPCI, MPI, PCS, PDI, SFMPQ and TSK) during the initial assessment session and completed this battery at home, returning it to the clinic on a subsequent appointment. Participants were not financially compensated for their participation. Consent for the assessment and for the use of their data in research was obtained by the clinician and recorded on the patient charts.

The second sample of chronic pain participants was drawn from a database generated from an epidemiological chronic pain survey (22). In this sample, individuals previously identified as chronic pain sufferers (ie, having pain for longer than six months) were contacted by telephone and were mailed the assessment battery, which was returned by mail. These individuals were compensated with $\$ 5.00$. Participants received the battery of measures in the same order as the clinic-referred participants (BAI, BDI, BHS, CPCI, MPI, PCS, PDI, SF-MPQ and TSK). Participants from this sample received two follow-up calls to ensure completion and to answer any questions. Of the 171 individuals with chronic pain identified from the epidemiological survey, 82 agreed to participate in the present research, 31 declined to participate, 27 were no longer experiencing pain, 20 were unreachable and 11 were no longer at the given phone number. Of the 82 identified participants, 62 returned the completed assessment measures.

\section{Descriptives}

\section{RESULTS}

Scores (mean \pm SD) for the 32 scales of the nine chronic pain measures are presented in Table 1. Data are presented for the total chronic pain sample, as well as separately for each of the clinic and survey samples.

Combination of the two samples

As shown in Table 1, the pain clinic sample had higher scores than the epidemiological survey sample on many of the measures used in the present study. Although this may raise concerns regarding the appropriateness of combining these two samples, 
separate analyses of each sample indicated that combining the two samples was appropriate. Discrepant scores do not necessarily indicate differing factor structures; it may be that even though the scores are different between the two groups, the underlying factor structures are similar. The clinic group was expected to produce more extreme scores because they were referred to a clinic for assessment and treatment. Preliminary factor analyses demonstrated similar factor structures in both samples. In general, each sample produced seven factors similar to one another and to the seven factors produced by combining the sample. Seven factors emerged that were consistent with the constructs of pain and disability, pain description, affective distress, support, positive coping strategies, negative coping strategies and activity. In addition to similar factor structures, the two samples produced similar factor loadings; the average difference in factor loadings between the two samples was 0.003 . Because of the similarities in factor structures and factor loadings, it was deemed appropriate to combine the two samples.

\section{Factor structure of individual measures}

The goal of the first set of data analyses was to derive the specific factor structures of each of the nine individual pain assessment measures for this sample. In a series of nine separate factor analyses, the items comprising each measure were submitted to an EFA. With these analyses, it was believed that EFA was more appropriate than CFA. There is no question that factor-analytic studies of each of the nine measures do exist. However, in reviewing these studies, considerable disagreement in factor structure was found, which may be due to differences in factor-analytic methodology and sample characteristics (eg, pain versus pain-free samples). For these reasons, EFA was deemed to be the appropriate analysis over CFA because there was no compelling empirical basis on which to precisely define the factors. Maximum likelihood was chosen to fit the common factor model to the data, and solutions were rotated using an oblique rotation (direct quartimin). To determine the appropriate number of factors for the measures, four procedures recommended by Fabrigar et al (7) were used in conjunction with one another. These factor-number procedures included examination of the scree plot of eigenvalues from the reduced correlation matrix, parallel analysis, the model fit procedure using RMSEA as the fit index and interpretability of the solution. These procedures are described in more detail below.

The final factor structures are presented in Table 2. These factors represent the 32 subscales that were used in a subsequent single EFA. (The complete set of analyses and results of the individual factor analyses for each of the nine measures is available from the first author.) For most of the chronic pain assessment measures examined, the structures that emerged were largely consistent with the results of previous factoranalytic studies. Factor analysis of the BAI yielded two factors, somatic complaints and subjective fear, which is consistent with previous factor-analytic studies of the measure (20,35). Other studies found conflicting results $(36,37)$. One factor, depressive symptomatology, emerged from the BDI factor analysis. Even though factor-analytic studies of the BDI yielded mixed results (31), the present finding was consistent with the way the BDI is used in clinical practice, as well as with previous factor-analytic research $(38,39)$. A three-factor solution (future uncertainty, expectations of failure and
TABLE 2

Subscales used in exploratory factor analysis of the nine chronic pain measures

\begin{tabular}{ll}
\hline Measure & Subscale(s) used \\
\hline BAI* $^{*}$ & Somatic complaints (items 1-3,6,8,12,13,15,19,21) \\
& Subjective fear (items 4,5,9,10,14,16,17) \\
BDI & Total BDI score \\
BHS* $^{*}$ & Future uncertainty (items 4,8,9,12,14,18) \\
& Expectations of failure (items 2,11,16,17,20) \\
CPCI & Expectations of success (items 1,13,15,19) \\
MPI & Oll eight CPCI subscales \\
PCS & Total PCS score \\
PDI & Total PDI score \\
SF-MPQ * & Affective descriptors (items 7,11,13-15) \\
& Acute sensory descriptors (items 2-4) \\
& Chronic sensory descriptors (items 1,8,10,12) \\
TSK & Total TSK score
\end{tabular}

*Indicates unique subscales created for the present study. BAI Beck Anxiety Inventory; BDI Beck Depression Inventory; BHS Beck Hopelessness Scale; $\mathrm{CPCl}$ Chronic Pain Coping Inventory; MPI Multidimensional Pain Inventory PCS Pain Catastrophizing Scale; PDI Pain Disability Index; SF-MPQ McGill Pain Questionnaire - Short Form; TSK Tampa Scale of Kinesiophobia

expectations of success) was concluded as best fitting the BHS data. This result is consistent with Dyce's factor-analytic study of the BHS (40). Other researchers proposed one- and twofactor solutions $(33,41)$. The CPCI factor analysis also produced results in agreement with previous studies. An eight-factor solution (guarding, resting, asking for assistance, relaxation, task persistence, exercise or stretching, seeking social support and coping self-statements) was selected as best fitting the data, which is consistent with the model proposed by its developers (18) and with the factor-analytic work of Hadjistavropoulos et al (42). A single factor representing disability was selected as best fitting the PDI data, which is consistent with a factor-analytic study performed by Chibnall and Tait (43). Other researchers concluded a two-factor solution (16). Finally, the factor analysis of the SF-MPQ yielded three factors: affective, acute sensory and chronic descriptors of pain. This finding is also consistent with the previous factor analyses of the measure (12). However, a two-factor structure has also been proposed (11).

For several measures, the factor structures that emerged were inconsistent with previously reported and hypothesized structures. The results of the MPI factor analysis were not easily interpretable, and none of the models examined conformed to the 12 -factor structure set out by its developers (44). This result may be due to problems with the design of the MPI itself (45). Given that the present results did not fit with the hypothesized MPI structure, and were not readily interpretable, three options were left. First, the factor structure that resulted from the analyses could be taken. However, this solution had no obvious conceptual meaning, and if that structure was included in subsequent analyses, it was not clear how those results could be interpreted. A second option was to treat the MPI as the original authors designed it. Although this option is not optimal, it would allow examination of how the MPI - as it is usually used - relates to the other measures used in the assessment of chronic pain. The third option would be to drop the MPI from all subsequent analyses. This is a valid option, 


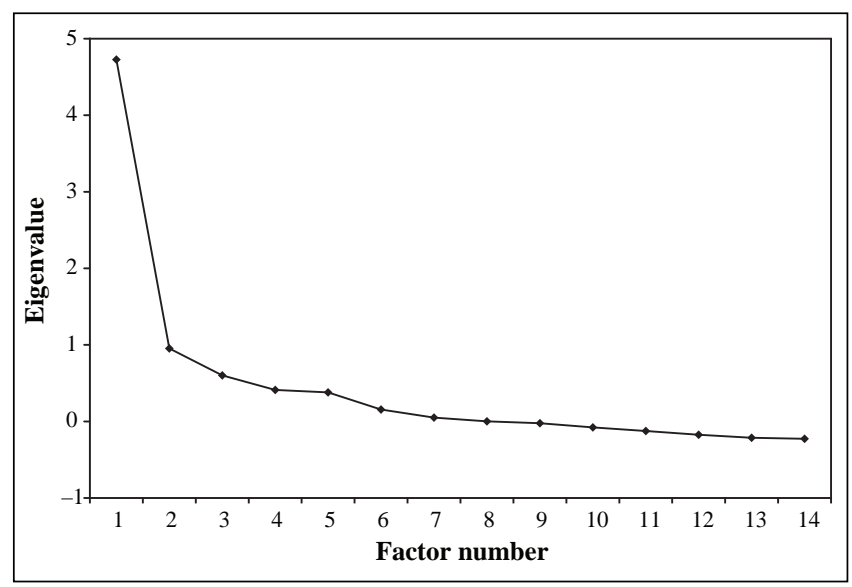

Figure 1) Scree plot of eigenvalues from factor analysis of the 32 subscales

but it may leave readers wondering how this popular measure relates to other measures used in chronic pain assessment. In considering these options, it was believed that the second provided information that was most useful to readers. Thus, the original MPI 12-factor structure was used in subsequent analyses. However, all MPI results should be interpreted with caution, because the factor structure of the measure is in question.

Problems also emerged with the PCS. A one-factor solution representing pain catastrophizing was concluded as best fitting the data. The PCS does yield a full-scale catastrophizing score, but it also produces three subscale scores (rumination, magnification and helplessness). The three-factor solution examined in the present study was not easily interpreted and, conceptually, did not make sense; items from each of the three scales (as set out by the test developer) did not load together. Other factoranalytic studies of the PCS have yielded two- (14) and threefactor $(13,15,46,47)$ solutions. Of note, the majority of these studies were conducted on nonpain samples.

With the TSK, three of the four factor-number procedures demonstrated consistent support for a three-factor solution. However, this model was not readily interpretable, and conceptually, it did not make sense. A one-factor model best accounted for the data. This result is not consistent with previous factor-analytic studies of the instrument (48-50); however, there is considerable disagreement among these studies as well. Thus, a one-factor solution reflecting one construct of the fear of movement, injury and reinjury was used in subsequent analyses. This is consistent with the clinical application of the TSK - the total score is generally used in interpretation of the measure.

\section{Factor structure of chronic pain scales}

The goal of the first set of data analyses was to derive the factor structures of each of the nine individual measures for this sample. These analyses yielded a total of 32 subscales from the nine chronic pain assessment measures. The second set of analyses investigated which subscales tapped into the same underlying factor and which subscales tapped into unique factors. Thus, the second part of the analyses moved to exploring the data at a subscale level, rather than at an item level. The EFA of the 32 subscales used the same factor-analytic procedures as the first set of analyses. The appropriate number of factors was determined using the same four factor-number procedures used in the above EFAs (scree plot analysis, parallel analysis, the model fit procedure using RMSEA as the fit index, and interpretability of the solution).

Scree plot: In scree plot analysis, the eigenvalues of the reduced correlation matrix are computed and then plotted in descending order. Each eigenvalue corresponds to the variance accounted for by a common factor. Thus, the appropriate number of factors corresponds to the last occasion when a substantial drop occurs in the magnitude of the eigenvalues.

The scree plot of eigenvalues from the reduced correlation matrix for the 32 subscales is presented in Figure 1. This scree plot is somewhat ambiguous, and the number of factors suggested by it is not clear. However, the substantial drops in the curve following the first, second and third eigenvalues suggest that a three-factor solution may be supported. There are also substantial dips in the curve following the sixth eigenvalue, suggesting that a six-factor solution is also possible. Therefore, based on this scree plot analysis, both three- and six-factor solutions are plausible.

Parallel analysis: The second factor-number procedure used was parallel analysis. Parallel analysis involves the comparison of eigenvalues from the reduced correlation matrix to eigenvalues one would expect to obtain from completely random data (ie, the predicted means of eigenvalues produced by repeated sets of random data). This procedure is based on the notion that sample eigenvalues greater than corresponding random data eigenvalues represent nonrandom effects (ie, latent factors). The number of factors in the model is determined by the number of eigenvalues that are greater than the eigenvalues predicted from random data. Two sets of expected values are generated in parallel analysis. The first represents the mean eigenvalue predicted from sets of random data. Observed values are compared with the mean, and any value greater than the mean is considered to be a nonrandom effect. However, using the mean eigenvalue may be too lenient. Although this criterion may identify nonrandom effects, some of the effects may be trivial and using the results could lead to overfactoring. Because of this issue, upper-bound values of the 95\% CI around the mean eigenvalue were also used in the present study. These upper-bound values represent a stricter criterion and may not be as prone to overfactoring as the use of the mean eigenvalue. The observed eigenvalues, the expected eigenvalues and the $95 \% \mathrm{CI}$ upper-bound eigenvalues corresponding to the EFA of the 32 chronic pain subscales are presented in Table 3. In the both the mean and upper-bound eigenvalues, the observed eigenvalue was greater than the expected value. Thus, by both criteria, a six-factor solution is supported.

Model fit: The third factor-number procedure used was the examination of model fit. RMSEA was used as an index of model fit. RMSEA is an estimate of the discrepancy between the model and the data per degree of freedom for the model. RMSEA values of 0.050 or lower indicate a good model fit, values between 0.051 and 0.080 indicate an acceptable model fit, values between 0.081 and 0.100 indicate a marginal model fit and a value higher than 0.100 indicates a poor model fit (51).

In the investigation of model fit, a series of models increasing in the number of factors is examined. Using RMSEA, the fit of the simplest model is examined first (one-factor), followed by the fit of a two-factor model, and so on. The optimum model 
TABLE 3

Observed eigenvalues, expected (random data) eigenvalues and upper-bound values of $95 \% \mathrm{Cls}$ from exploratory factor analysis of the 32 subscales

\begin{tabular}{|c|c|c|c|}
\hline Factor & Observed & Expected & $\begin{array}{l}95 \% \text { upper- } \\
\text { bound value }\end{array}$ \\
\hline 1 & 8.839 & 1.383 & 1.537 \\
\hline 2 & 4.050 & 1.210 & 1.333 \\
\hline 3 & 1.700 & 1.080 & 1.193 \\
\hline 4 & 1.517 & 0.975 & 1.070 \\
\hline 5 & 1.199 & 0.882 & 0.969 \\
\hline 6 & 1.060 & 0.799 & 0.893 \\
\hline 7 & 0.653 & 0.718 & 0.785 \\
\hline 8 & 0.467 & 0.653 & 0.719 \\
\hline 9 & 0.440 & 0.585 & 0.645 \\
\hline 10 & 0.387 & 0.523 & 0.592 \\
\hline 11 & 0.300 & 0.461 & 0.519 \\
\hline 12 & 0.283 & 0.399 & 0.465 \\
\hline 13 & 0.229 & 0.342 & 0.394 \\
\hline 14 & 0.156 & 0.286 & 0.335 \\
\hline 15 & 0.141 & 0.236 & 0.285 \\
\hline 16 & 0.131 & 0.183 & 0.230 \\
\hline 17 & 0.096 & 0.136 & 0.185 \\
\hline 18 & 0.002 & 0.092 & 0.146 \\
\hline 19 & 0.000 & 0.042 & 0.094 \\
\hline 20 & -0.029 & -0.000 & 0.045 \\
\hline 21 & -0.033 & -0.042 & -0.003 \\
\hline 22 & -0.064 & -0.080 & -0.043 \\
\hline 23 & -0.086 & -0.119 & -0.086 \\
\hline 24 & -0.102 & -0.159 & -0.124 \\
\hline 25 & -0.118 & -0.198 & -0.169 \\
\hline 26 & -0.131 & -0.233 & -0.209 \\
\hline 27 & -0.139 & -0.267 & -0.239 \\
\hline 28 & -0.179 & -0.303 & -0.276 \\
\hline 29 & -0.196 & -0.334 & -0.314 \\
\hline 30 & -0.205 & -0.371 & -0.349 \\
\hline 31 & -0.213 & -0.407 & -0.382 \\
\hline
\end{tabular}

is one in which having one fewer factor produces a much poorer model fit, and having one additional factor does not increase model fit substantially. In addition, the magnitude of improvement in RMSEA between models was also considered. Although small changes in RMSEA from one model to another do indicate improvement in model fit, choosing a model based on relatively minor improvements in fit can result in overfactoring. The ultimate goal is to arrive at the most parsimonious model that adequately accounts for the data. RMSEA values and corresponding CIs were compared for a series models, from one to eight factors. RMSEA values and 90\% CIs for the eight solutions are presented in Table 4.

With an RMSEA value greater than 0.100 , the one-factor model demonstrated a poor model fit. The two-factor model decreased RMSEA by 0.026 and represented a substantial improvement, and moved model fit into the marginal range. The three-factor model also improved model fit somewhat, with a 0.010 decrease in RMSEA, representing a marginal model fit. A decrease of 0.009 in RMSEA from the three-factor model to the four-factor model represented some improvement in model fit, moving it into the acceptable range. The five-factor model demonstrated a 0.012 RMSEA decrease. The six-factor
TABLE 4

Root mean square error of approximation (RMSEA) values and corresponding $90 \% \mathrm{Cls}$ from exploratory factor analysis of the 32 subscales

\begin{tabular}{lcc}
\hline Number of factors & RMSEA & $\mathbf{9 0 \%} \mathbf{~ C l}$ \\
\hline 1 & 0.119 & $0.111-0.127$ \\
2 & 0.093 & $0.084-0.101$ \\
3 & 0.083 & $0.074-0.092$ \\
4 & 0.074 & $0.064-0.084$ \\
5 & 0.062 & $0.051-0.088$ \\
6 & 0.051 & $0.051-0.074$ \\
7 & 0.036 & $0.013-0.052$ \\
8 & 0.031 & $0.000-0.049$ \\
\hline
\end{tabular}

model also improved model fit modestly, with a 0.011 decrease in RMSEA, moving model fit into the good range. RMSEA was also improved with the seven-factor solution $(0.015$ decrease in RMSEA). However, the CI for the seven-factor solution noticeably increased, indicating the possibility of overfactoring. The eight-factor model improved model fit only slightly. Based on this model fit assessment, six- and sevenfactor solutions were supported.

Interpretability of solutions: The final factor number procedure used was the interpretability of the model. When deciding on the appropriate number of factors, factor interpretability is an important criterion to consider. Even if all other criteria indicate a particular number of factors, the model is of little value to the researcher if it is not easily interpretable or conceptually sensible. Thus, one must always consider relevant theory and previous research when determining the appropriate number of factors. To this point, the factor-number procedures used in examining factor structure underlying the 32 subscales seemed to support a six- or seven-factor solution. The interpretability of these two solutions was examined.

To identify the items that primarily loaded on each factor, a few general guidelines were used. Factor loadings that equalled or exceeded 0.40 were considered to be substantial loadings, and loadings between 0.20 and 0.39 were considered to be marginal loadings. Values less than 0.20 were considered to be low. The six-factor solution yielded five interpretable factors, which appeared to represent dimensions of affective distress, coping, support, pain and activities. However, the sixth factor comprised two scales, and it did not make sense conceptually.

The seven-factor solution yielded seven interpretable factors. Factor loadings and communalities are presented in Table 5. The first factor appeared to represent pain and disability, with the MPI interference and pain severity subscales, and the PDI all loading substantially. The second factor appeared to represent positive coping strategies, and comprised four CPCI subscales: coping self-statements, relaxation, seeking social support, and exercise or stretching. All of these strategies are generally encouraged in chronic pain treatment. The third factor appeared to represent support, with MPI solicitous responses, MPI support, MPI distracting responses and CPCI asking for assistance all loading substantially. The fourth factor that emerged was interpreted as pain description, with all three subscales of the SF-MPQ (affective, acute sensory and chronic sensory) loading substantially. With the fifth factor, three subscales loaded substantially (CPCI guarding, TSK and CPCI resting). This factor appeared to represent fear of movement 
TABLE 5

Pattern matrix of rotated seven-factor solution for exploratory factor analysis of the 32 subscales

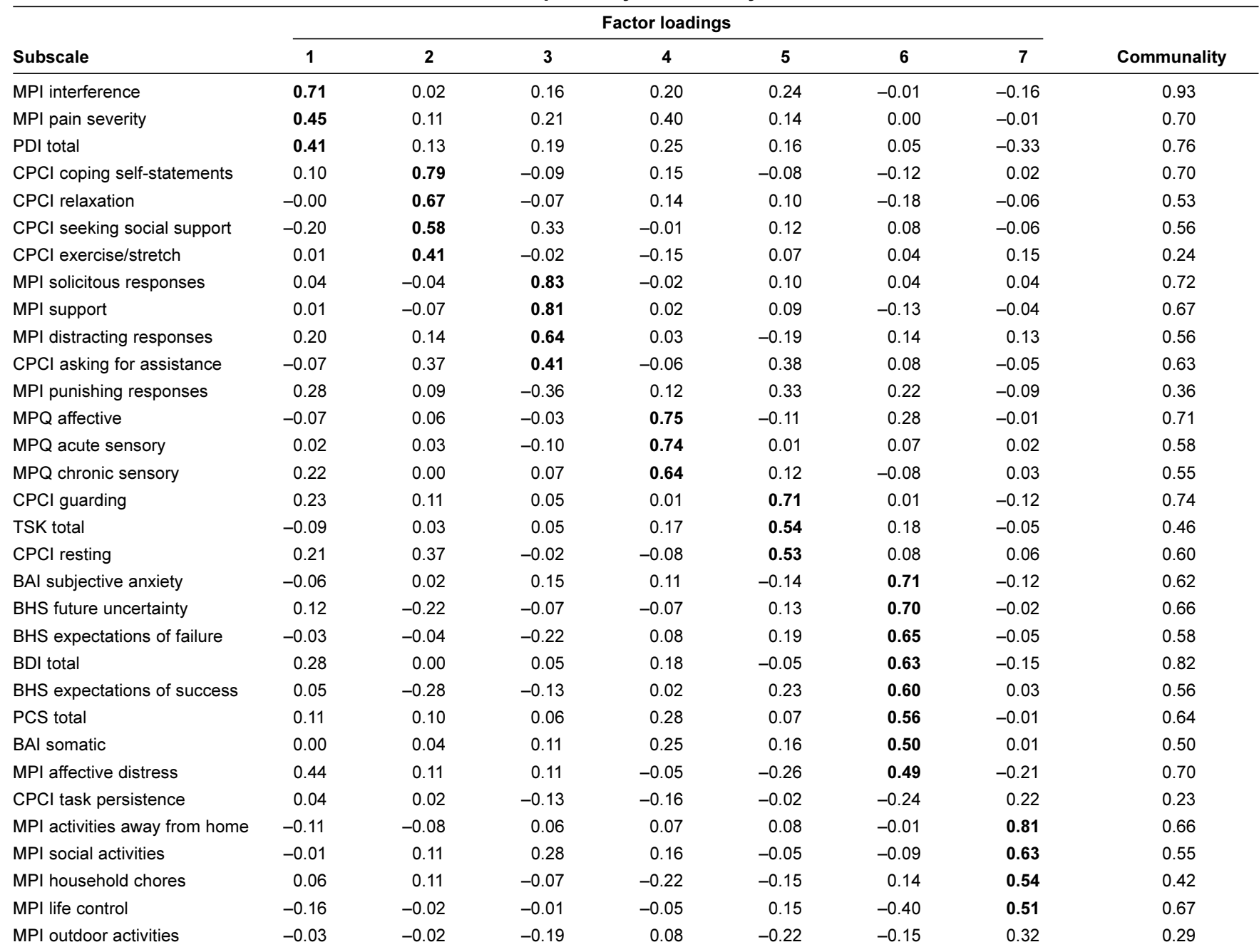

Values in bold indicate subscales with substantial loadings ( $\geq 0.40$ ). BAI Beck Anxiety Inventory; BDI Beck Depression Inventory; BHS Beck Hopelessness Scale CPCI Chronic Pain Coping Inventory; MPI Multidimensional Pain Inventory; MPQ McGill Pain Questionnaire; PCS Pain Catastrophizing Scale; PDI Pain Disability Index; TSK Tampa Scale of Kinesiophobia

and the use of negative coping strategies. The sixth factor appeared to represent affective distress, with nine measures loading substantially: BAI subjective anxiety, BHS future uncertainty, BHS expectations of failure, BDI, BHS expectations of success, PCS, BAI somatic complaints and MPI affective distress. Finally, the seventh factor was interpreted as representing activity, with three of the MPI activities subscales (activities away from home, social activities and household chores) and MPI life control loading. Only two subscales failed to load substantially: MPI punishing responses (which marginally loaded on the support factor) and CPCI task persistence.

Based on this examination of the interpretability of the sixand seven-factor solutions, a seven-factor model was selected as representing the most interpretable solution. Seven conceptually sensible factors emerged, all with substantial factor loadings. This seven-factor solution accounted for $59 \%$ of the total variance.

\section{DISCUSSION}

The underlying structure of the 32 empirically derived chronic pain subscales was examined, and results yielded seven interpretable factors, each representing a different chronic pain construct: pain and disability, pain description, affective distress, positive coping, negative coping, support and activity. All of the factors that emerged represent constructs that have been highlighted as important measurement targets for therapy within the chronic pain literature.

Four of the seven factors are also supported by the two earlier factor-analytic studies of chronic pain measures. The functional capacity factor identified in the factor analyses of measures of Mikail et al (2) and De Gagne et al (3) is very similar to the present study's pain and disability factor. Their factor included similar subscales, including a disability index and the MPI interference and pain severity subscales. Mikail et al and De Gagne et al also reported a pain description factor, which, similar to the present study, was composed of MPQ subscales. An affective distress component also emerged in these two previous factoranalytic studies, and comprised a number of depression and anxiety subscales. The support factor from the present study was also previously reported. Mikail et al and De Gagne et al identified a support factor, which consisted of subscales indexing different types of social support. 
Although a number of the factors found in the present study replicated previous research, several novel factors emerged. Whereas Mikail et al (2) reported a single coping factor composed of a number of different types of coping strategies, we found that coping divided into two different types of coping strategies: positive coping and negative coping. Jensen et al (18) suggested this delineation of coping strategies, but it has not been reported in any of the factor-analytic studies to date. Activity also emerged as a distinct factor in the present study. This, too, represents a novel factor that has not emerged in any of the previous factor-analytic studies of pain measures. These findings likely reflect our use of more comprehensive chronic pain measures compared with those used in the two previous factor-analytic studies. The pain assessment literature has advanced since these two studies, and the present study included a number of recently developed measures (eg, CPCI, PCS, TSK) to reflect these advances. The differences in factor structure may also be due to differences in factor-analytic methodologies, as well as to differences in samples.

The present study joins only two published studies examining and detailing specific factors underlying chronic pain assessment measures. While earlier studies attempted to identify and understand these factors, they were limited. The two other factor-analytic studies, similar to the present study in design and study population, provided a good foundation on which to further examine these pain assessment factors $(2,3)$. The present study improved on these studies in a number of important ways. The two existing factor-analytic studies used each measure and its subscales as indicated by the developers. The present study improved on these studies by using empirically derived chronic pain factor subscales. Rather than accept the structures reported by the developers, the present study used a more robust methodology with factor structures that were verified in an earlier set of analyses in the same sample. In addition, for all factor analyses, we used multiple criteria to determine the appropriate number of factors.

The present results have clinical implications. A comprehensive chronic pain assessment battery should reflect the multiple aspects of the pain experience (52). However, in the chronic pain literature, there is no consensus regarding choice of measures (2), and little research has attempted to define the optimal composition of a prototypical chronic pain assessment battery. With replication, the present results will have implications for multifaceted treatment programs, in which a small number of measures can be selected to best assess each of the seven factors that emerged in the present study. For example, one could reduce the battery of nine measures used in the present study by choosing one or two scales from each of the seven factors. This reduction in measures has the potential to lighten the assessment load for both patients and clinicians, and to ensure that important dimensions of the chronic pain experience are measured in a parsimonious fashion. Using only a select group of measures, clinicians would be able to assess multiple aspects of the chronic pain experience efficiently. At the same time, the item burden on patients would be reduced substantially, and consequently, clinicians would have fewer data to analyze and interpret.

The present study has several limitations. The sample was drawn from two sources - a group of individuals with chronic pain referred to an outpatient pain clinic and a group of individuals with chronic pain identified through an epidemiological survey. By increasing the heterogeneity of our sample, the results of the present study were intended to be generalizable to a larger group of individuals with chronic pain. Our analyses demonstrated that combining the two samples was appropriate. However, the generalizability of the results has not been verified. It is possible that in using factor structures derived using the present study's sample, we created sample-specific factor structures for each of the nine measures, thereby limiting the generalizability of the results. We do know, however, that the structures used in our factor analysis of chronic pain measures are plausible representations for our sample. Clearly, further research is needed to determine whether these results are generalizable to other pain populations.

An additional concern may be the size of the present study's sample. There is no effective heuristic for determining sample size when conducting an EFA. Guidelines do exist (eg, five participants per measured variable, or ratios of 10 to 1 ); however, a number of researchers noted that such guidelines are not sufficiently sensitive to a variety of important characteristics of the data $(7,53,54)$. Adequate sample size is not a function of the number of measured variables per se, but by the extent to which factors are overdetermined, and by the level of the communalities of the obtained variables. When an EFA is performed on variables with low communalities, substantial distortion of results can occur. An overdetermined factor refers to a factor that is represented by at least three or four measured variables. Under good conditions (communalities of 0.70 or higher, four to five variables for each factor), a sample size of 100 may be adequate for EFA. Under conditions of moderate communalities ( 0.40 to 0.70 ) and moderate overdetermination of factors, a sample of 200 or more is advisable (7). In the present study, four of the seven factors were clearly overdetermined, and the remaining three factors were moderately overdetermined. The average communality of the obtained variables was 0.60 , which is closer to the upper boundary of the moderate communality range than at the low end. These results suggest relatively good conditions for our factor analysis, so it is unlikely that sample size has seriously distorted the results.

The results of the present analyses are convincing, but preliminary. Some of the factors that emerged represented novel dimensions not identified in the two previous factor-analytic studies. Future research should replicate and further examine these seven factors underlying chronic pain measures in both general chronic pain samples and specific pain samples.

\section{REFERENCES}

1. Turk DC, Okifuji A. Psychological factors in chronic pain: Evolution and revolution. J Consult Clin Psychol 2002;70:678-90.

2. Mikail SF, DuBreuil SC, D'Eon JL. A comparative analysis of measures used in the assessment of chronic pain patients. Psychol Assess 1993;5:117-20.

3. De Gagne TA, Mikail SF, D'Eon JL. Confirmatory factor analysis of a 4-factor model of chronic pain evaluation. Pain 1995;60:195-202.

4. Beck AT, Ward CH, Mendelson M, Mock J, Erbaugh J. An inventory for measuring depression. Arch Gen Psychiatry 1961;4:561-71.

5. Melzack R. The McGill Pain Questionnaire: Major properties and scoring methods. Pain 1975;1:277-99.

6. Rudy TE. Multiaxial Assessment of Pain: Multidimensional Pain Inventory Computer Program User's Manual, Version 2.1. Pittsburgh: University of Pittsburgh, 1989.

7. Fabrigar LR, Wegener DT, MacCallum RC, Strahan EJ. Evaluating the use of exploratory factor analysis in psychological research. Psychol Methods 1999;4:272-99. 
8. Zwick WR, Velicer WF. Factors influencing four rules for determining the number of components to retain. Multivariate Behav Res 1982;17:253-69.

9. Zwick WR, Velicer WF. Comparison of five rules for determining the number of components to retain. Psychol Bull 1986;99:432-42.

10. Melzack R. The short-form McGill Pain Questionnaire. Pain 1987;30:191-7.

11. Wright KD, Asmundson GJ, McCreary DR. Factorial validity of the short-form McGill pain questionnaire (SF-MPQ). Eur J Pain 2001;5:279-84

12. Burckhardt CS, Bjelle A. A Swedish version of the short-form McGill Pain Questionnaire. Scand J Rheumatol 1994;23:77-81.

13. Sullivan MJL, Bishop SR, Pivik J. The Pain Catastrophizing Scale: Development and validation. Psychol Assess 1995;7:524-532.

14. Osman A, Barrios FX, Kopper BA, Hauptmann W, Jones J, O'Neill E. Factor structure, reliability, and validity of the Pain Catastrophizing Scale. J Behav Med 1997;20:589-605.

15. Osman A, Barrios FX, Gutierrez PM, Kopper BA, Merrifield T, Grittmann L. The Pain Catastrophizing Scale: Further psychometric evaluation with adult samples. J Behav Med 2000;23:351-65.

16. Tait RC, Pollard CA, Margolis RB, Duckro PN. The Pain Disability Index: Psychometric and validity data. Arch Phys Med Rehabil 1987;68:438-41.

17. Kori SH, Miller RP, Todd DD. Kinesiophobia: A new view of chronic pain behavior. Pain Manage 1990;35-43.

18. Jensen MP, Turner JA, Romano JM, Strom SE. The Chronic Pain Coping Inventory: Development and preliminary validation. Pain 1995;60:203-16

19. Beck AT, Weissman A, Lester D, Trexler L. The measurement of pessimism: The hopelessness scale. J Consult Clin Psychol 1974;42:861-5.

20. Beck AT, Epstein N, Brown G, Steer RA. An inventory for measuring clinical anxiety: Psychometric properties. J Consult Clin Psychol 1988;56:893-7.

21. Becker N, Thomsen AB, Olsen AK, Sjøgren P, Bech P, Eriksen J. Pain epidemiology and health related quality of life in chronic nonmalignant pain patients referred to a Danish multidisciplinary pain center. Pain 1997;73:393-400.

22. Tripp DA, VanDenKerkhof EG, McAlister M. Prevalence and determinants of pain and pain-related disability in urban and rural settings in southeastern Ontario. Pain Res Manage 2006;11:225-33.

23. Von Korff M, Ormel J, Keefe FJ, Dworkin SF. Grading the severity of chronic pain. Pain 1992;50:133-49.

24. Statistics Canada. Community highlights for Kingston. $<$ http://www12.statcan.ca/english/profil01/CP01/Details/Page.cfm?L ang $=\mathrm{E} \& \mathrm{Geo} 1=\mathrm{CSD} \&$ Code $1=3510010 \& \mathrm{Geo} 2=\mathrm{PR} \& \mathrm{Code} 2=35 \& \mathrm{D}$ ata $=$ Count $\&$ Search Text $=$ kingston $\&$ Search Type $=$ Begins $\&$ SearchPR $=35 \& B 1=$ All\&Custom $=>($ Version current at March 28, 2008).

25. Grafton KV, Foster NE, Wright CC. Test-retest reliability of the Short-Form McGill Pain Questionnaire: Assessment of intraclass correlation coefficients and limits of agreement in patients with osteoarthritis. Clin J Pain 2005;21:73-82

26. Tait RC, Chibnall JT, Krause S. The Pain Disability Index: Psychometric properties. Pain 1990;40:171-82.

27. Roelofs J, Goubert L, Peters ML, Vlaeyen JW, Crombez G. The Tampa Scale for Kinesiophobia: Further examination of psychometric properties in patients with chronic low back pain and fibromyalgia. Eur J Pain 2004;8:495-502.

28. Swinkels-Meewisse EJ, Swinkels RA, Verbeek AL, Vlaeyen JW, Oostendorp RA. Psychometric properties of the Tampa Scale for kinesiophobia and the Fear-Avoidance Beliefs Questionnaire in acute low back pain. Man Ther 2003;8:29-36.

29. Swinkels-Meewisse IE, Roelofs J, Verbeek AL, Oostendorp RA, Vlaeyen JW. Fear of movement/(re)injury, disability and participation in acute low back pain. Pain 2003;105:371-9.

30. Wittink H, Turk DC, Carr DB, Sukiennik A, Rogers W. Comparison of the redundancy, reliability, and responsiveness to change among SF-36, Oswestry Disability Index, and Multidimensional Pain Inventory. Clin J Pain 2004;20:133-42.

31. Beck AT, Steer RA, Garbin MG. Psychometric properties of the Beck Depression Inventory: Twenty-five years of evaluation. Clin Psychol Rev 1988;8:77-100.

32. Durham TW. Norms, reliability, and item analysis of the hopelessness scale in general, psychiatric, forensic psychiatric, and college populations. J Clin Psychol 1982;38:597-600.

33. Steed L. Further validity and reliability evidence for Beck Hopelessness Scale scores in a nonclinical sample. Educ Psychol Meas 2001;61:303-16

34. Osman A, Kopper BA, Barrios FX, Osman JR, Wade T. The Beck Anxiety Inventory: Re-examination of factor structure and psychometric properties. J Clin Psychol 1997;53:7-14.

35. Steer RA, Kumar G, Ranieri WF, Beck AT. Use of the Beck Anxiety Inventory with adolescent psychiatric outpatients. Psychol Rep 1995;76:459-65

36. Borden JW, Peterson DR, Jackson EA. The Beck Anxiety Inventory in nonclinical samples: Initial psychometric properties. J Psychopathol Behav Assess 1991;13:345-56.

37. Osman A, Barrios FX, Aukes D, Osman JR, Markway K. The Beck Anxiety Inventory: Psychometric properties in a community population. J Psychopathol Behav Assess 1993;15:287-97.

38. Clark DC, Gibbons RD, Fawcett J, Aagesen CA, Sellers D. Unbiased criteria for the severity of depression in alcoholic inpatients. J Nerv Ment Dis 1985;173:482-7.

39. Novy DM, Nelson DV, Berry LA, Averill PM. What does the Beck Depression Inventory measure in chronic pain? A reappraisal. Pain 1995;61:261-70.

40. Dyce JA. Factor structure of the Beck Hopelessness Scale. J Clin Psychol 1996;52:555-8.

41. Chang EC, D'Zurilla TJ, Maydeu-Olivares A. Assessing the dimensionality of optimism and pessimism using a multimeasure approach. Cognit Ther Res 1994;18:143-60.

42. Hadjistavropoulos HD, MacLeod FK, Asmundson GJ. Validation of the Chronic Pain Coping Inventory. Pain 1999;80:471-81.

43. Chibnall JT, Tait RC. The Pain Disability Index: Factor structure and normative data. Arch Phys Med Rehabil 1994;75:1082-6.

44. Kerns RD, Turk DC, Rudy TE. The West Haven-Yale Multidimensional Pain Inventory (WHYMPI). Pain 1985;23:345-56.

45. Deisinger J, Cassisi JE, Lofland KR, Cole P, Bruehl S. An examination of the psychometric structure of the Multidimensional Pain Inventory. J Clin Psychol 2001;57:765-83.

46. D'Eon JL, Harris CA, Ellis JA. Testing factorial validity and gender invariance of the pain catastrophizing scale. J Behav Med 2004;27:361-72

47. Van Damme S, Crombez G, Bijttebier P, Goubert L, Van Houdenhove B. A confirmatory factor analysis of the Pain Catastrophizing Scale: Invariant factor structure across clinical and non-clinical populations. Pain 2002;96:319-24.

48. Geisser ME, Haig AJ, Theisen E. Activity avoidance and function in persons with chronic back pain. J Occup Rehabil 2000;10:215-27.

49. Goubert L, Crombez G, Van Damme S, Vlaeyen JW, Bijttebier P, Roelofs J. Confirmatory factor analysis of the Tampa Scale for Kinesiophobia: Invariant two-factor model across low back pain patients and fibromyalgia patients. Clin J Pain 2004;20:103-10.

50. Vlaeyen JWS, Kole-Snijders AMJ, Rotteveel AM, Ruesink R, Heuts PHTG. The role of fear of movement/(re)injury in pain disability. J Occup Rehabil 1995;5:235-52.

51. Browne MW, Cudeck R. Alternative ways of assessing model fit. Sociol Methods Res 1992;21:230-58.

52. Williams RC. Toward a set of reliable and valid measures for chronic pain assessment and outcome research. Pain 1988;353:239-51.

53. MacCallum RC, Widaman KF, Zhang S, Hong S. Sample size in factor analysis. Psychol Methods 1999;4:84-99.

54. Velicer WF, Fava JL. Affects of variable and subject sampling on factor pattern recovery. Psychol Methods 1998;3:231-51. 


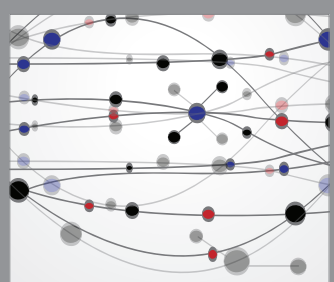

The Scientific World Journal
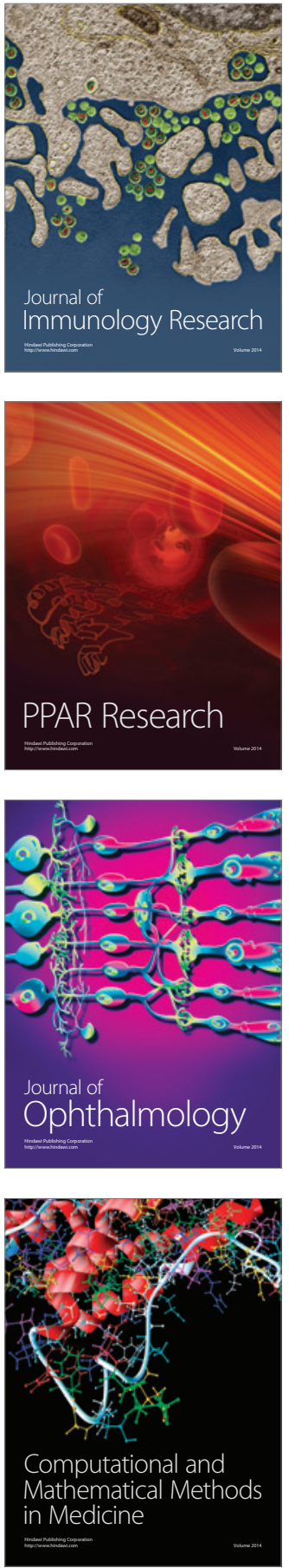

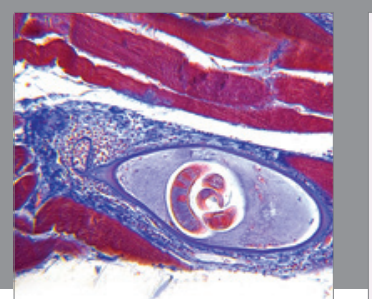

Gastroenterology Research and Practice

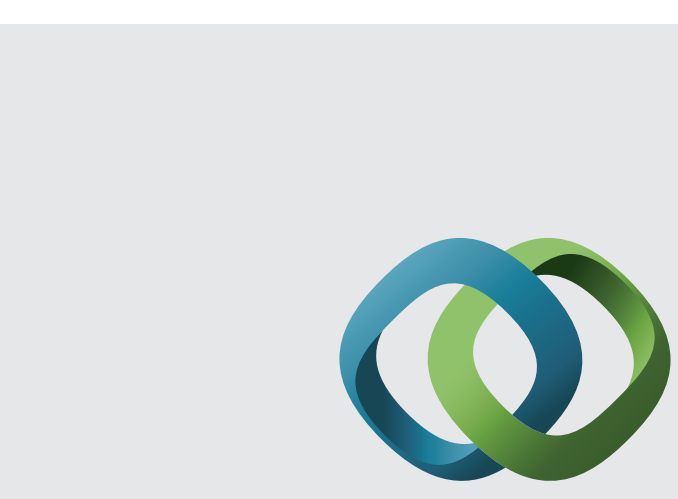

\section{Hindawi}

Submit your manuscripts at

http://www.hindawi.com
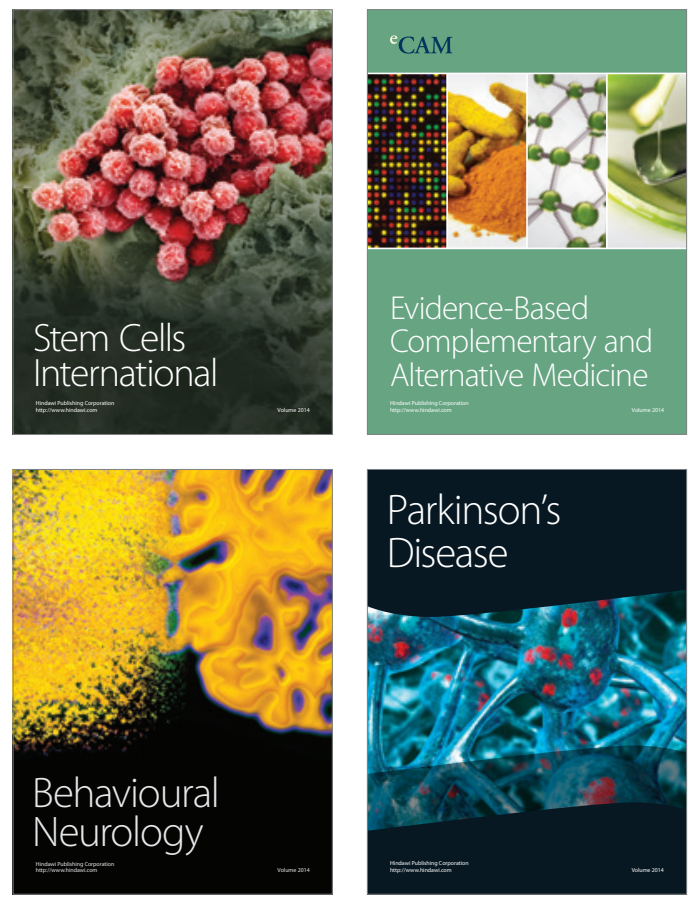
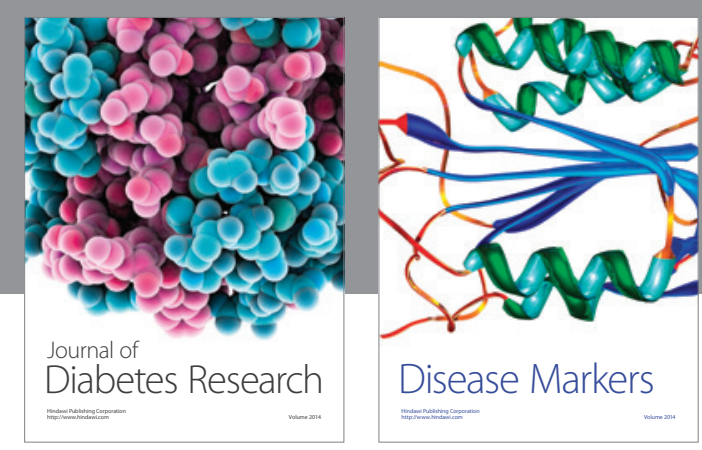

Disease Markers
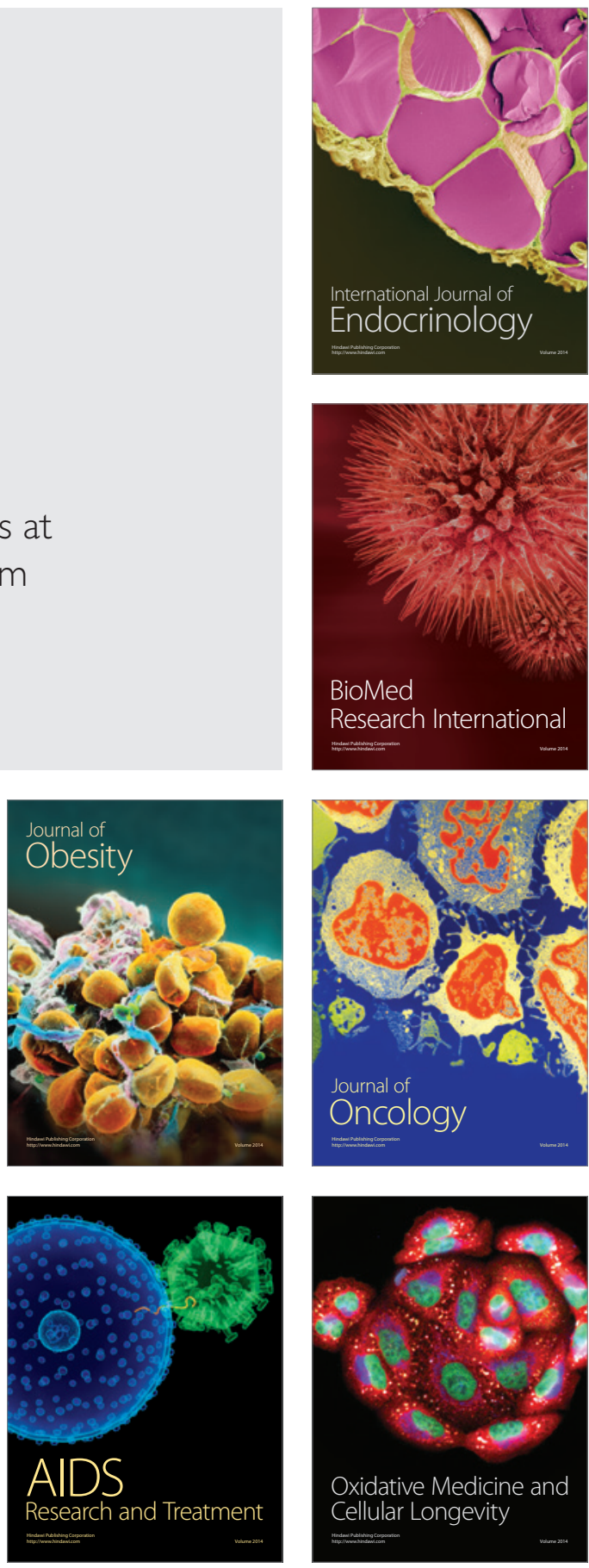